\title{
On the Remainder Term of Some Bivariate Approximation Formulas Based on Linear and Positive Operators
}

\author{
DAN BĂRBOSU*
}

\begin{abstract}
The paper is a survey concerning representations for the remainder term of Bernstein-Schurer-Stancu and respectively Stancu (based on factorial powers) bivariate approximation formulas, using bivariate divided differences. As particular cases the remainder terms of bivariate Bernstein-Stancu, Schurer and classical Bernstein bivariate approximation formulas are obtained. Finally, one presents some mean value properties, similar to those of the remainder term of classical Bernstein univariate approximation formula.
\end{abstract}

Keywords: Bernstein-Schurer-Stancu bivariate operator, Stancu bivariate operator, Bivariate divided difference, Bivariate approximation formula, Remainder term

2010 Mathematics Subject Classification: 41A80, 41A36.

\section{INTRODUCTION}

Denote by $\mathbb{N}$ the set of positive integers and $\mathbb{N}_{0}=\mathbb{N} \cup\{0\}$. Let $p \in \mathbb{N}$ be given and $\alpha, \beta$ be real parameters satisfying the condition $0 \leq \alpha \leq \beta$. The Bernstein-Schurer-Stancu operator is defined [6] for any $m \in \mathbb{N}$, any $f \in C[0,1+p]$ and any $x \in[0,1+p]$ by:

$$
\widetilde{S}_{m, p}^{(\alpha, \beta)}(f ; x)=\sum_{k=0}^{m+p} \widetilde{p}_{m, k}(x) f\left(\frac{k+\alpha}{m+\beta}\right)
$$

where

$$
\widetilde{p}_{m, k}(x)=\left(\begin{array}{c}
m+p \\
k
\end{array}\right) x^{k}(1-x)^{m+p-k},
$$

are the fundamental Schurer polynomials [33].

Note that the multiparameter operator (1.1) contains as particular cases the Schurer operator [33] (for $\alpha=\beta=0$ ), the Bernstein-Stancu operator [36] (for $p=0$ ) and of course the classical Bernstein operator [18] (for $p=0$ and $\alpha=\beta=0$ ). Many of its approximation properties were investigated in [9].

Let $p, q \in \mathbb{N}_{0}$ be given and let $\alpha, \beta, \gamma, \delta$ be real parameters such that $0 \leq \alpha \leq \beta, 0 \leq \gamma \leq \delta$.

Using the method of parametric extensions [21], [5], in [7] was introduced the BernsteinSchurer-Stancu bivariate operator, given by

$$
\widetilde{S}_{m, p, n, q}^{(\alpha, \beta, \gamma, \delta)}(f ; x, y)=\sum_{k=0}^{m+p} \sum_{j=0}^{n+q} \widetilde{p}_{m, k}(x) \widetilde{p}_{n, j}(y) f\left(\frac{k+\alpha}{m+\beta}, \frac{j+\gamma}{n+\delta}\right)
$$

while in [8] was considered its GBS associated operator, given by

Received: July 10, 2018; In revised form: September 10, 2018; Accepted: October 1, 2018

*Corresponding author: D. Bărbosu; barbosudan@yahoo.com

DOI: $10.33205 / \mathrm{cma} .442151$ 


$$
\begin{aligned}
& \widetilde{U}_{m, p, n, q}^{(\alpha, \beta, \gamma)}(f ; x, y)= \\
& \sum_{k=0}^{m+p} \sum_{j=0}^{n+q} \widetilde{p}_{m, k}(x) \widetilde{q}_{n, j}(y)\left\{f\left(\frac{k+\alpha}{m+\beta}, y\right)+f\left(x, \frac{j+\gamma}{n+\delta}\right)-f\left(\frac{k+\alpha}{m+\beta}, \frac{j+\gamma}{n+\delta}\right)\right\} .
\end{aligned}
$$

Approximation properties of operator (1.3) and (1.4) were established in [7], [9], [13].

In Section 2 we recall the results from $[12,13]$ regarding the remainder term of the bivariate Bernstein-Schurer-Stancu bivariate approximation formula. At end of this section, we discuss about the remainder term of GBS Bernstein approximation formula [11].

Suppose $\alpha$ is a non-negative parameter which may depends only of $m \in \mathbb{N}$. In 1968, Stancu [36] introduced the operator $P^{\langle\alpha\rangle}: C[0,1] \rightarrow C[0,1]$ given by

$$
P_{m}^{\langle\alpha\rangle}(f ; x)=\sum_{k=0}^{m} p_{m, k}^{\langle\alpha\rangle}(x) f\left(\frac{k}{m}\right),
$$

where $p_{n, k}^{\langle\alpha\rangle}(x)$ are Stancu's fundamental polynomials expressed by means of factorial power $t^{[m, h]}=t(t-h) \ldots(t-(m-1) h), t^{[0, h]}=1$, by

$$
p_{m, k}^{\langle\alpha\rangle}(x)=\left(\begin{array}{c}
m \\
k
\end{array}\right) \frac{x^{[k,-\alpha]}(1-x)^{[m-k,-\alpha]}}{1^{[m,-\alpha]}},
$$

for any $x \in[0,1], m \in \mathbb{N}$ and $k \in\{0,1, \ldots, m\}$.

The operator (1.5) is the Stancu operator (or Stancu operator based on factorial power).

Let $m, n \in \mathbb{N}$ be given and let $\alpha=\alpha(m), \beta=\beta(n)$ be real parameters. Using the method of parametric extension in $[25,26]$ was obtained the Stancu operator and its GBS-Stancu associated operator, given respectively by

$$
P_{m, n}^{\langle\alpha, \beta\rangle}(f ; x, y)=\sum_{k=0}^{m} \sum_{j=0}^{n} p_{m, k}^{\langle\alpha\rangle}(x) p_{n, j}^{\langle\beta\rangle}(y) f\left(\frac{k}{m}, \frac{i}{n}\right)
$$

and

$$
U_{m, n}^{\langle\alpha, \beta\rangle}(f ; x, y)=\sum_{k=0}^{m} \sum_{j=0}^{n} p_{m, k}^{\langle\alpha\rangle}(x) p_{n, j}^{\langle\beta\rangle}(y)\left\{f\left(\frac{i}{m}, y\right)+f\left(x, \frac{i}{n}\right)-f\left(\frac{i}{m}, \frac{i}{n}\right)\right\} .
$$

The corresponding approximation formulas were studied in the recently papers $[25,26]$ due to Miclăuş, where the remainders of mentioned formula were expressed using bivariate divided differences.

Coming back to the classical Bernstein operator $B_{m}: C[0,1] \rightarrow C[0,1]$ given by

$$
B_{m}(f ; x)=\sum_{k=0}^{m} p_{m, k} f\left(\frac{k}{m}\right)
$$

for any $f \in C[0,1], x \in[0,1]$ and $m \in \mathbb{N}$, recall that Aramă [4], proved that the remainder term of the univariate Bernstein approximation formula

$$
f=B_{m}(f)+R_{m}(f)
$$

can be represented under the form 


$$
R_{m}(f ; x)=-\frac{x(1-x)}{m}\left[\xi_{1}, \xi_{2}, \xi_{3} ; f\right]
$$

for any $x \in[0,1]$, where $0 \leq \xi_{1}<\xi_{2}<\xi_{3} \leq 1$.

In Section 3 we will present analogous results for the remainder terms of bivariate BernsteinSchurer-Stancu and respectively Stancu bivariate approximation formulas.

\section{THE REMAINDER TERM OF THE BIVARIATE BERNSTEIN-SCHURER-STANCU APPROXIMATION FORMULA}

We start by recalling some results regarding the divided differences which will be used in the paper. Suppose that $I \subset \mathbb{R}$ is an interval of the real axis and $x_{1}, x_{2} \in I$ such that $x_{1} \neq x_{2}$. The divided difference of $f$ with respect the distinct knots $x_{1}, x_{2}$ is defined by

$$
\left[x_{1}, x_{2} ; f\right]=\frac{f\left(x_{2}\right)-f\left(x_{1}\right)}{x_{2}-x_{1}} .
$$

If $x_{0}, x_{1}, \ldots, x_{n} \in I$ are distinct knots and $f: I \rightarrow \mathbb{R}$ is given, then the $n$-th order divided difference of $f$ with respect the mentioned knots is defined by the recurrence relation

$$
\left[x_{0}, x_{1}, \ldots, x_{m} ; f\right]=\frac{\left[x_{1}, \ldots, x_{m} ; f\right]-\left[x_{0}, \ldots, x_{m-1} ; f\right]}{x_{m}-x_{0}} .
$$

Note that the divided differences were intensively studied by Popoviciu [30]. Interesting properties of the divided differences were obtained by Ionescu [22] and Ivan [23].

Let $I, J \subset \mathbb{R}$ be intervals, $f: I \times J \rightarrow \mathbb{R}$ be bounded and $\left(x_{1}, y_{1}\right),\left(x_{2}, y_{2}\right) \in I \times J$ such that $x_{1} \neq x_{2}, y_{1} \neq y_{2}$. The bivariate divided difference of $f$ with respect the knots $\left(x_{1}, y_{1}\right),\left(x_{2}, y_{2}\right)$ is defined [10] by

$$
\left[\begin{array}{l}
x_{1}, x_{2} \\
y_{1}, y_{2}
\end{array} ; f\right]=\frac{f\left(x_{2}, y_{2}\right)-f\left(x_{1}, y_{2}\right)-f\left(x_{2}, y_{1}\right)+f\left(x_{1}, y_{1}\right)}{\left(x_{2}-x_{1}\right)\left(y_{2}-y_{1}\right)} .
$$

Other equivalent definitions were given in the monographs by Ionescu [22] and Ivan [23].

In the definition of the bivariate divided difference the number of abscissas is in general not equal with the number of coordinates. For example (see [10]), we have that

$$
\left[\begin{array}{c}
x_{1}, x_{2} \\
y_{1}
\end{array} ; f\right]=\frac{f\left(x_{2}, y_{1}\right)-f\left(x_{1}, y_{1}\right)}{x_{2}-x_{1}}
$$

and

$$
\left[\begin{array}{cc}
x_{1}, x_{2}, x_{3} & ; f \\
y_{1} & ;
\end{array}\right]=\frac{1}{x_{3}-x_{2}}\left(\left[\begin{array}{cc}
x_{2}, x_{3} & ; f \\
y_{1} & ;
\end{array}\right]-\left[\begin{array}{cc}
x_{1}, x_{2} & ; f \\
y_{1} & ;
\end{array}\right]\right)
$$

where $x_{1}, x_{2}, x_{3} \in I$ are distinct.

For the bivariate divided difference with respect the distinct knots $\left(x_{i}, y_{j}\right) \in I \times J(i=$ $\overline{0, m}, j=\overline{0, n}$ ) the following recurrence formula [10] 
(2.17)

$$
\left[\begin{array}{l}
x_{0}, x_{1}, \ldots, x_{m} \\
y_{0}, y_{1}, \ldots, y_{n}
\end{array} ; f\right]=
$$

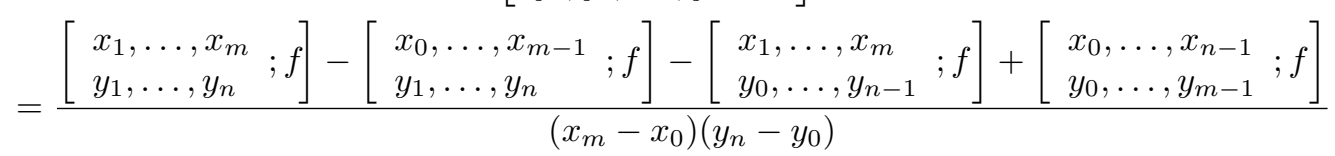

holds.

Using the above mentioned properties of the bivariate divided differences, in [13] was established the following.

Theorem 2.1. The remainder term of the bivariate Bernstein-Schurer-Stancu approximation formula

$$
f=\widetilde{S}_{m, p, n, q}^{(\alpha, \beta, \gamma, \delta)}(f)+\widetilde{R}_{m, p, n, q}^{(\alpha, \beta, \gamma)}(f)
$$

can be represented under the form

$$
\widetilde{R}_{m, p, n, q}^{(\alpha, \beta, \gamma)}(f ; x, y)=S_{1}+S_{2}+S_{3},
$$

where

$$
\begin{aligned}
& S_{1}=\frac{(\beta-p) x-\alpha}{m+\beta} \sum_{k=0}^{m+p} \sum_{j=0}^{n+q} \widetilde{p}_{m, k}(x) \widetilde{p}_{n, j}(y)\left[\begin{array}{c}
x, \frac{k+\alpha}{m+\beta} \\
\frac{j+\gamma}{n+\delta}
\end{array} ; f\right] \\
& -\frac{x(1-x)(m+p)}{(m+\beta)^{2}} \sum_{k=0}^{m+p-1} \sum_{j=0}^{n+q} \widetilde{p}_{m-1, k}(x) \widetilde{p}_{n, j}(y)\left[\begin{array}{c}
x, \frac{k+\alpha}{m+\beta}, \frac{k+\alpha+1}{m+\beta} \\
\frac{j+\gamma}{n+\delta}
\end{array} ; f\right] \text {; } \\
& S_{2}=\frac{(\delta-q) y-\gamma}{n+\delta} \sum_{k=0}^{m+p} \sum_{j=0}^{n+q} \widetilde{p}_{m, k}(x) \widetilde{p}_{n, j}(y)\left[\begin{array}{c}
\frac{k+\alpha}{m+\beta} \\
y, \frac{j+\gamma}{n+\delta}
\end{array} ; f\right] \\
& -\frac{y(1-y)(n+q)}{(n+\delta)^{2}} \sum_{k=0}^{m+p} \sum_{j=0}^{n+q-1} \widetilde{p}_{m, k}(x) \widetilde{p}_{n-1, j}(y)\left[\begin{array}{c}
\frac{k+\alpha}{m+\beta} \\
y, \frac{j+\gamma}{n+\delta}, \frac{j+\gamma+1}{n+\delta}
\end{array} ; f\right] \text {; }
\end{aligned}
$$

$$
\begin{aligned}
S_{3}= & x y(1-x)(1-y) \frac{(m+p)(n+q)}{(m+\beta)^{2}(n+\delta)^{2}} \\
& \left.\times \sum_{k=0}^{m+p-1} \sum_{j=0}^{n+q-1} \widetilde{p}_{m-1, k}(x) \widetilde{p}_{n-1, j}(y)\left[\begin{array}{c}
x, \frac{k+\alpha}{m+\beta}, \frac{k+\alpha+1}{m+\beta} \\
y, \frac{j+\gamma}{n+\delta}, \frac{j+\gamma+1}{n+\delta}
\end{array}\right] f\right] \\
& -\frac{m+p}{(m+\beta)^{2}(n+\delta)} x(1-x)\{(\delta-q) y-\gamma\} \\
& \times \sum_{k=0}^{m+p-1} \sum_{j=0}^{n+q} \widetilde{p}_{m-1, k}(x) \widetilde{p}_{n, j}(y)\left[\begin{array}{c}
x, \frac{k+\alpha}{m+\beta}, \frac{k+\alpha+1}{m+\beta} \\
y, \frac{j+\gamma}{n+\delta}
\end{array}\right] \\
& -\frac{n+q}{(m+\beta)(n+\delta)^{2}} y(1-y)\{(\beta-p) x-\alpha\}
\end{aligned}
$$




$$
\begin{aligned}
& \times \sum_{k=0}^{m+p} \sum_{j=0}^{n+q-1} \widetilde{p}_{m, k}(x) \widetilde{p}_{n-1, j}(y)\left[\begin{array}{c}
x, \frac{k+\alpha}{m+\beta} \\
y, \frac{j+\gamma}{n+\delta}, \frac{j+\gamma+1}{n+\delta}
\end{array} ; f\right] \\
& +\frac{1}{(m+\beta)(n+\gamma)}\{(\beta-p) x-\alpha\}\{(\delta-q) y-\gamma\} \\
& \times \sum_{k=0}^{m+p} \sum_{j=0}^{n+q} \widetilde{p}_{m, k}(x) \widetilde{p}_{n, j}(y)\left[\begin{array}{c}
x, \frac{k+\alpha}{m+\beta} \\
y, \frac{j+\gamma}{n+\delta}
\end{array}\right] .
\end{aligned}
$$

Remark 2.1. For $\alpha=\beta=\gamma=\delta=0$ one obtains that the Bernstein-Schurer-Stancu operator reduces to the Schurer bivariate operator, given by

$$
\widetilde{B}_{m, p, n, q}(f ; x, y)=\sum_{k=0}^{m+p} \sum_{j=0}^{n+q} \widetilde{p}_{m, k}(x) \widetilde{p}_{n, j}(y) f\left(\frac{k}{m+p}, \frac{j}{n+q}\right) .
$$

In this case we get the Schurer bivariate approximation formula

$$
f=\widetilde{B}_{m, p, n, q}(f)+\widetilde{R}_{m, p, n, q}(f) .
$$

Applying the Theorem 2.1 for $\alpha=\beta=\gamma=\delta=0$, it follows

Corollary 2.1. The remainder term of the Schurer bivariate approximation formula (2.18) can be represented under the form:

$$
\widetilde{R}_{m, p, n, q}(f)=S_{1}+S_{2}+S_{3}
$$

where

$$
\begin{aligned}
S_{1}= & \left.-\frac{p x}{m} \sum_{k=0}^{m+p} \sum_{j=0}^{n+q} \widetilde{p}_{m, k}(x) \widetilde{p}_{n, j}(y)\left[\begin{array}{c}
x, \frac{k}{m} \\
\frac{j}{n}
\end{array}\right] f\right] \\
& -\frac{x(1-x)(m+p)}{m^{2}} \sum_{k=0}^{m+p-1} \sum_{j=0}^{n+q} \widetilde{p}_{m-1, k}(x) \widetilde{p}_{n, j}(y)\left[\begin{array}{c}
x, \frac{k}{m}, \frac{k+1}{m} \\
\frac{j}{n}
\end{array}\right] ; f
\end{aligned}
$$

$$
\begin{aligned}
S_{2}= & -\frac{q y}{n} \sum_{k=0}^{m+p} \sum_{j=0}^{n+q} \widetilde{p}_{m, k}(x) \widetilde{p}_{n, j}(y)\left[\begin{array}{c}
\frac{k}{m} \\
y, \frac{j}{n}
\end{array} ; f\right] \\
& -\frac{y(1-y)(n+q)}{n^{2}} \sum_{k=0}^{m+p} \sum_{j=0}^{n+q-1} \widetilde{p}_{m, k}(x) \widetilde{p}_{n-1, j}(y)\left[\begin{array}{c}
\frac{k}{m} \\
y, \frac{j}{n}, \frac{j+1}{n}
\end{array} ; f\right] ;
\end{aligned}
$$




$$
\begin{aligned}
S_{3}= & \left.\frac{x y(1-x)(1-y)}{m n} \sum_{k=0}^{m+p-1} \sum_{j=0}^{n+q-1} \widetilde{p}_{m-1, k}(x) \widetilde{p}_{n-1, j}(y)\left[\begin{array}{c}
x, \frac{k}{m}, \frac{k+1}{m} \\
y, \frac{j}{n}, \frac{j+1}{n}
\end{array}\right] f\right] \\
& +\frac{(m+p) q}{m^{2} n} x y(1-x) \sum_{k=0}^{m+p-1} \sum_{j=0}^{n+q} \widetilde{p}_{m-1, k}(x) \widetilde{p}_{n, j}(y)\left[\begin{array}{c}
x, \frac{k}{m}, \frac{k+1}{m} \\
y, \frac{j}{n}
\end{array} ; f\right] \\
& +\frac{(n+q) p}{m n^{2}} x y(1-y) \sum_{k=0}^{m+p} \sum_{j=0}^{n+q-1} \widetilde{p}_{m, k}(x) \widetilde{p}_{n-1, j}(y)\left[\begin{array}{c}
x, \frac{k}{m} \\
y, \frac{j}{n}, \frac{j+1}{n}
\end{array} ; f\right] \\
& +\frac{p q}{m n} x y \sum_{k=0}^{m+p} \sum_{j=0}^{n+q} \widetilde{p}_{m, k}(x) \widetilde{p}_{n, j}(y)\left[\begin{array}{c}
x, \frac{k}{m} \\
y, \frac{j}{n}
\end{array} ; f\right] .
\end{aligned}
$$

Remark 2.2. For $p=q=0$, the bivariate Bernstein-Schurer-Stancu bivariate operator (2.25) reduces to the bivariate Bernstein-Stancu operator, given by

$$
S_{m, n}^{(\alpha, \beta, \gamma, \delta)}(f ; x, y)=\sum_{k=0}^{m} \sum_{j=0}^{n} p_{m, k}(x) p_{n, j}(y) f\left(\frac{k+\alpha}{m+\beta}, \frac{j+\gamma}{n+\delta}\right) .
$$

Consequently it follows the Bernstein-Stancu bivariate approximation formula

$$
f=S_{m, n}^{(\alpha, \beta, \gamma, \delta)}(f)+R_{m, n}^{(\alpha, \beta, \gamma, \delta)}(f) .
$$

Regarding the remainder term of (2.29), applying the Theorem 2.1 for $p=q=0$, it follows:

Corollary 2.2. The remainder term of (2.29) can be expressed under the form:

$$
R_{m, n}^{(\alpha, \beta, \gamma, \delta)}(f ; x, y)=S_{1}+S_{2}+S_{3},
$$

where

$$
\begin{aligned}
S_{1} & \left.=\frac{\beta x-\alpha}{m+\beta} \sum_{k=0}^{m} \sum_{j=0}^{n} p_{m, k}(x) p_{n, j}(y)\left[\begin{array}{c}
x, \frac{k+\alpha}{m+\beta} \\
\frac{j+\gamma}{n+\delta}
\end{array}\right] f\right] \\
- & \frac{m x(1-x)}{(m+\beta)^{2}} \sum_{k=0}^{m-1} \sum_{j=0}^{n} p_{m-1, k}(x) p_{n, j}(y)\left[\begin{array}{c}
x, \frac{k+\alpha}{m+\beta}, \frac{k+\alpha+1}{m+\beta} \\
\frac{j+\gamma}{n+\delta}
\end{array} ; f\right] ; \\
S_{2} & =\frac{\delta y-\gamma}{n+\delta} \sum_{k=0}^{m} \sum_{j=0}^{n} p_{m, k}(x) p_{n, j}(y)\left[\begin{array}{c}
\frac{k+\alpha}{m+\beta} \\
y, \frac{j+\gamma}{n+\delta}
\end{array} ; f\right] \\
& -\frac{n y(1-y)}{(n+\delta)^{2}} \sum_{k=0}^{m} \sum_{j=0}^{n-1} p_{m, k}(x) p_{n-1, j}(y)\left[\begin{array}{c}
\frac{k+\alpha}{m+\beta} \\
y, \frac{j+\gamma}{n+\delta}, \frac{j+\gamma+1}{n+\delta}
\end{array}\right] ;
\end{aligned}
$$




$$
\begin{aligned}
S_{3}= & \frac{m n x y(1-x)(1-y)}{(m+\beta)^{2}(n+\delta)^{2}} \sum_{k=0}^{m-1} \sum_{j=0}^{n-1} p_{m-1, k}(x) p_{n-1, j}(y)\left[\begin{array}{c}
x, \frac{k+\alpha}{m+\beta}, \frac{k+\alpha+1}{m+\beta} \\
y, \frac{j+\gamma}{n+\delta}, \frac{j+\gamma+1}{n+\delta}
\end{array} ; f\right] \\
& -\frac{m}{(m+\beta)^{2}(n+\delta)} x(1-x)(\delta y-\gamma) \sum_{k=0}^{m-1} \sum_{j=0}^{n} p_{m-1, k}(x) p_{n, j}(y)\left[\begin{array}{c}
x, \frac{k+\alpha}{m+\beta}, \frac{k+\alpha+1}{m+\beta} \\
y, \frac{j+\gamma}{n+\delta}
\end{array} ; f\right] \\
& -\frac{n}{(m+\beta)(n+\delta)^{2}} y(1-y)(\beta x-\alpha) \sum_{k=0}^{m} \sum_{j=0}^{n-1} p_{m, k}(x) p_{n-1, j}(y)\left[\begin{array}{c}
x, \frac{k+\alpha}{m+\beta} \\
y, \frac{j+\gamma}{n+\delta}, \frac{j+\gamma+1}{n+\delta}
\end{array} ; f\right] \\
& +\frac{1}{(m+\beta)(n+\gamma)}(\beta x-\alpha)(\delta y-\gamma) \sum_{k=0}^{m} \sum_{j=0}^{n} p_{m, k}(x) p_{n, j}(y)\left[\begin{array}{c}
x, \frac{k+\alpha}{m+\beta} \\
y, \frac{j+\gamma}{n+\delta}
\end{array}\right] .
\end{aligned}
$$

Remark 2.3. When $p=q=0, \alpha=\beta=\gamma=\delta=0$, one obtains the classical Bernstein bivariate operator, namely

$$
B_{m, n}(f ; x, y)=\sum_{k=0}^{m} \sum_{j=0}^{n} p_{m, k}(x) p_{n, j}(y) f\left(\frac{k}{m}, \frac{j}{n}\right) .
$$

The associated Bernstein bivariate approximation formula is

$$
f=B_{m, n}(f)+R_{m, n}(f),
$$

and, as consequence of Theorem 2.1, for its remainder term we have the following:

Corollary 2.3. The remainder term of (2.35) can be represented under the form:

$$
R_{m, n}(f ; x, y)=S_{1}+S_{2}+S_{3},
$$

where

$$
\begin{aligned}
& S_{1}=-\frac{x(1-x)}{m} \sum_{k=0}^{m-1} \sum_{j=0}^{n} p_{m-1, k}(x) p_{n, j}(y)\left[\begin{array}{c}
x, \frac{k}{m}, \frac{k+1}{m} \\
\frac{j}{n}
\end{array} ; f\right], \\
& S_{2}=-\frac{y(1-y)}{n} \sum_{k=0}^{m} \sum_{j=0}^{n-1} p_{m, k}(x) p_{n-1, j}(y)\left[\begin{array}{c}
\frac{k}{m} \\
y, \frac{j}{n}, \frac{j+1}{n}
\end{array} ; f\right], \\
& S_{3}=\frac{x y(1-x)(1-y)}{m n} \sum_{k=0}^{m-1} \sum_{j=0}^{n-1} p_{m-1, k}(x) p_{n-1, j}(y)\left[\begin{array}{l}
x, \frac{k}{m}, \frac{k+1}{m} \\
y, \frac{j}{n}, \frac{j+1}{n}
\end{array} ; f\right] .
\end{aligned}
$$

Applying the mean value theorem for bivariate divided differences, in [13] was proved the following:

Theorem 2.2. Let be $f \in C^{(2,2)}([0,1+p] \times[0,1+q])$. Then there exists a constant $M$ depending on $f, \alpha, \beta, \gamma, \delta$, such that for any $(x, y) \in[0,1] \times[0,1]$ and any $m, n \in \mathbb{N}$, the following

$$
\left|\widetilde{R}_{m, p, n, q}^{(\alpha, \beta, \gamma)}(f ; x, y)\right| \leq\left(\frac{9 m+p}{8 m^{2}}+\frac{9 n+q}{8 n^{2}}+\frac{(9 m+p)(9 n+q)}{64 m^{2} n^{2}}\right) M
$$

holds. 
Remark 2.4. From (2.40) similar estimations for the remainders of Schurer, Bernstein-Stancu and respectively Bernstein bivariate approximation formulas can be derived.

Let now $U_{m, n}$ be the GBS Bernstein operator, given by

$$
U_{m, p}(f ; x, y)=\sum_{k=0}^{m} \sum_{j=0}^{n} p_{m, k}(x) p_{n, j}(y)\left\{f\left(\frac{k}{m}, y\right)+f\left(x, \frac{j}{n}\right)-f\left(\frac{k}{m}, \frac{j}{n}\right)\right\}
$$

(it is obtained from the operator (1.4), for $\alpha=\beta=\gamma=\delta=p=q=0$ ).

Considering the GBS Bernstein approximation formula

$$
f=U_{m, n}(f)+R_{m, n}(f)
$$

in [12] we proved the following:

Theorem 2.3. The remainder term of (2.42) can be expressed under the form

$$
R_{m, n}(f ; x, y)=\frac{x y(1-x)(1-y)}{m n} \sum_{k=0}^{m-1} \sum_{j=0}^{n-1} p_{m-1, k}(x) p_{n-1, j}(y)\left[\begin{array}{l}
x, \frac{k}{m}, \frac{k+1}{m} \\
y, \frac{j}{n}, \frac{j+1}{n}
\end{array} ; f\right] .
$$

Applying the mean value theorem for, bivariate divided differences, in [12] was proved

Theorem 2.4. Suppose $f \in C^{(1,1)}([0,1] \times[0,1])$ is a function for which exist $\frac{\partial^{4} f}{\partial x^{2} \partial y^{2}}$ on $[0,1] \times[0,1]$, bounded on $[0,1] \times[0,1]$. Then, the following inequalities

$$
\left|R_{m, n}(f ; x, y)\right| \leq \frac{x y(1-x)(1-y)}{4 m n} M(f) \leq \frac{1}{64 m n} M(f)
$$

hold, for any $(x, y) \in[0,1] \times[0,1]$, where

$$
M(f)=\sup _{(x, y) \in[0,1] \times[0,1]}\left|\frac{\partial^{4} f}{\partial x^{2} \partial y^{2}}(x, y)\right|
$$

3. MEAN VALUE RESULTS FOR THE REMAINDER TERM OF SOME BIVARIATE APPROXIMATION FORMULAS

We start by recalling some notions regarding the convex functions of higher order.

In his Ph. Thesis (June 1933) Popoviciu [30] introduced the notion of $m$-th order convexity for univariate real valued functions as follow:

Definition 3.1. Let $I \subseteq \mathbb{R}$ be an interval. The function $f \in \mathbb{R}^{I}$ is $m$-th order convex (nonconcave, polynomial, non-convex, concave) on $I$ if and only if for each $(m+2)$ distinct points $x_{1}, x_{2}, \ldots, x_{m+2} \in I$ one of the following inequalities hold true

$$
\left[x_{1}, x_{2}, \ldots, x_{m+2} ; f\right]>0(\geq 0,=0, \leq 0,<0),
$$

where the brackets denote divided difference.

Note that monotonous strictly increasing is convex of 1-th order wheil a strictly convex function in the usual sense is convex of 2-th order. Among many others, in [30] was established the following:

Theorem 3.5. Suppose $a, b \in \mathbb{R}$ such that $a<b$ and $A \in C^{\#}[a, b]$ is a linear functional defined on $[a, b]$. Suppose that

(i) $A(1)=A(x)=\ldots=A\left(x^{m}\right)=0, A\left(x^{m+1}\right) \neq 0$; 
(ii) $A(g) \neq 0$ for each $g \in C[a, b]$ convex of $m$-th order. that

Then, for each $f \in C[a, b]$ there exist $(m+2)$ distinct points $a \leq \xi_{1}<\xi_{2}<\ldots<\xi_{m+2} \leq b$ such

$$
A(f)=K\left[\xi_{1}, \xi_{2}, \ldots, \xi_{m+2} ; f\right],
$$

where $K$ is a constant independently on $f$.

Applying Popoviciu's theorem, Aramă [4] established the following mean value result for the remainder term of Bernstein univariate approximation formula.

Theorem 3.6. Let $B_{m}: C[0,1] \rightarrow C[0,1]$ be the Bernstein operator. The remainder term of the Bernstein univariate approximation formula

$$
f=B_{m}(f)+R_{m}(f)
$$

can be expressed under the form

$$
R_{m}(f ; x)=-\frac{x(1-x)}{m}\left[\xi_{1}, \xi_{2}, \xi_{3} ; f\right]
$$

where $0 \leq \xi_{1}<\xi_{2}<\xi_{3} \leq 1$.

Suppose $I, J \subseteq \mathbb{R}$ are compact intervals, $C(I \times J)=\left\{f \in \mathbb{R}^{I \times J} \mid f\right.$ continuos on $\left.I \times J\right\}, C^{\#}(I \times$ $J)=\{A: C(I \times J) \rightarrow \mathbb{R} \mid A-$ linear $\}$.

Our goal is to obtain a representation of a linear functional $A \in C^{\#}(I \times J)$ associated to functions $f \in C[a, b]$ which satisfy some special conditions.

Let $D \subseteq \mathbb{R}^{2}$ be a convex set, $m, n \in \mathbb{N},\left(x_{i}, y_{i}\right) \in D$ for each $i=\overline{1, m}$, $j=\overline{1, n}$ and $f \in R^{D}$ be a bivariate bounded real valued functions. Recall that the bivariate divided difference of $f$ on the distinct points $\left(x_{i}, y_{i}\right)$ is given by

$$
\left[\begin{array}{l}
x_{1}, \ldots, x_{m} \\
y_{1}, \ldots, y_{n}
\end{array} ; f\right]=\left[y_{1}, \ldots, y_{n} ;\left[x_{1}, \ldots, x_{m} ; f\right]\right] .
$$

The notion of $(m, n)$ - th order bivariate divided difference was introduced by Popoviciu [30] and then studied by many others (see for example [10,11], [22,23]).

The notion of $(m, n)-t h$ order bivariate convex function was introduced by Popoviciu [30] in the following:

Definition 3.2. The function $f \in \mathbb{R}^{D}$ is $(m, n)$ - th order convex (non concave, polynomial, non convex, concave) if and only if for each distinct points $\left(x_{i}, y_{i}\right)(i \in\{1, \ldots, m+2\}, j \in\{1, \ldots, n+2\}$, one of the following inequalities

$$
\left[\begin{array}{l}
x_{1}, \ldots, x_{m+2} \\
y_{1}, \ldots, y_{n+2}
\end{array} ; f\right]>0(\geq 0,=0, \leq 0,<0)
$$

holds.

Example 3.1. A monotonous increasing function $f \in \mathbb{R}^{D}$ is convex of $(0,0)$-th order, a strictly convex function in the usual sense is convex of $(1,1)$-th order in the sense of definition (3.47), etc.

The analogous of Theorem 3.2 for the bivariate case is the following 
Theorem 3.7. Suppose $I, J \subseteq \mathbb{R}$ are compact intervals and denote

$$
C^{\#}(I, J)=\{A: C(I \times J) \rightarrow \mathbb{R} \mid A-\text { linear }\} .
$$

If the linear functional $A$ satisfies the conditions:

(i) $A\left(x^{i} y^{j}\right)=0,(\forall) i \in\{0,1, \ldots, m\},(\forall) j \in\{0,1, \ldots, n\}$;

(ii) $A\left(x^{m+1} y^{n+1}\right) \neq 0$;

(iii) $A(g) \neq 0$ for each $g \in C(I \times J)$ convex of $(m, n)$ - th order, then, for any $f \in C(I \times J)$ there exist the distinct points $\left(\xi_{i} \eta_{j}\right)(i \in\{1, \ldots, m+2\}, j \in\{1, \ldots, n+2\}$,$) such that$

$$
A(f)=K\left[\begin{array}{c}
\xi_{1}, \ldots, \xi_{m+2} \\
\eta_{1}, \ldots, \eta_{n+2}
\end{array} ; f\right]
$$

where $K$ is a constant independently of $f$.

Proof. From the hypothesis (iii) it follows that $A(g) \neq 0$ for each $g \in C(I \times J)$ concave of $(m, n)$-th order. Let $f \in C(I \times J)$ be arbitrarily chosen and let $g \in C(I \times J)$ be given by

$$
g(x, y)=f(x, y)-\frac{A(f)}{A\left(x^{m+1} g^{n+1}\right)} x^{m+1} g^{n+1},
$$

for any $(x, y) \in I \times J$.

From (3.53) it follows that $A(g)=0$, and consequently $g$ is not convex and not concave of $(m, n)$-th order on $I \times J$. According to Definition 3.2, there exist the distinct points $\left(\xi_{i} \eta_{j}\right) \in I \times J$ $(i \in\{1, \ldots, m+2\}, j \in\{1, \ldots, n+2\}$,$) such that$

$$
\left[\begin{array}{c}
\xi_{1}, \ldots, \xi_{m+2} \\
\eta_{1}, \ldots, \eta_{n+2}
\end{array} ; f\right]=0
$$

Taking the linearity of bivariate divided difference into account, from (3.53) and (3.54) are obtains

$$
\left[\begin{array}{c}
\xi_{1}, \xi_{2}, \ldots, \xi_{m+2} \\
\eta_{1}, \eta_{2}, \ldots, \eta_{n+2}
\end{array} ; f\right]-\frac{A(f)}{A\left(x^{m+1} g^{n+1}\right)}\left[\begin{array}{c}
\xi_{1}, \ldots, \xi_{m+2} \\
\eta_{1}, \ldots, \eta_{n+2}
\end{array} ; x^{m+1} g^{n+1}\right]=0 .
$$

But it is well known (see [10] or [29]) that

$$
\left[\begin{array}{c}
\xi_{1}, \ldots, \xi_{m+2} \\
\eta_{1}, \ldots, \eta_{n+2}
\end{array} ; x^{m+1} g^{n+1}\right]=1 .
$$

Taking (3.56) into account, from (3.55) one arrives to the desired result with $K=A\left(x^{m+1} g^{n+1}\right)$.

Application 3.1. Consider the bivariate Bernstein approximation formula

$$
f=B_{m, n}(f)+R_{m, n}(f),
$$

where $B_{m, n}: C([0,1] \times[0,1]) \rightarrow C([0,1] \times[0,1])$ is the Bernstein bivariate operator, i.e.

$$
B_{m, n}(f ; x, y)=\sum_{k=0}^{m} \sum_{j=0}^{n} p_{m, k}(x) p_{n, j}(y) f\left(\frac{k}{m}, \frac{i}{n}\right) .
$$


It is well known (see for example [17]) that $B_{m, n}(f ; x, y)>f(x, y)$ for any $(x, y) \in[0,1] \times[0,1]$ and any $f \in C([0,1] \times[0,1])$, convex of $(1,1)$-th order on $[0,1] \times[0,1]$.

Let $\left(x_{0}, y_{0}\right) \in[0,1] \times[0,1]$ be arbitrarily chosen and define the functional $A \in C^{\#}([0,1] \times[0,1])$ by

$$
A(f)=f\left(x_{0}, y_{0}\right)-B_{m, n}\left(f ; x_{0}, y_{0}\right) .
$$

From the properties of the bivariate Bernstein operator it follows

$$
\begin{aligned}
& A\left(x_{0}^{i} y_{0}^{j}\right)=0, \text { for } i \in\{0,1\}, j \in\{0,1\} \\
& A\left(x_{0}^{2} y_{0}^{2}\right) \neq 0 \\
& A(g) \neq 0 \text { for any }(1,1) \text {-th order convex function. }
\end{aligned}
$$

Applying the Theorem 3.2, there exist the distinct point $\left(\xi_{i} \eta_{j}\right) \in[0,1] \times[0,1], i \in\{1,2,3\}, j \in$ $\{1,2,3\}$ such that

$$
A(f)=A\left(x_{0}^{2} y_{0}^{2}\right)\left[\begin{array}{c}
\xi_{1}, \xi_{2}, \xi_{3} \\
\eta_{1}, \eta_{2}, \eta_{3}
\end{array} ; f\right] .
$$

But $A(f)=R_{m, n}(f)$ and taking into account that

$$
A\left(x_{0}^{2} y_{0}^{2}\right)=R_{m, n}\left(x_{0}^{2} y_{0}^{2}\right)=\frac{x_{0} y_{0}\left(1-x_{0}\right)\left(1-y_{0}\right)}{m n},
$$

from (3.64) one arrives to

$$
R_{m, n}\left(f ; x_{0}, y_{0}\right)=\frac{x_{0} y_{0}\left(1-x_{0}\right)\left(1-y_{0}\right)}{m n}\left[\begin{array}{l}
\xi_{1}, \xi_{2}, \xi_{3} \\
\eta_{1}, \eta_{2}, \eta_{3}
\end{array} ; f\right] .
$$

Because $\left(x_{0}, y_{0}\right) \in[0,1] \times[0,1]$ was arbitrarily chosen, it follows (3.64) holds in each point $(x, y) \in[0,1] \times[0,1]$

Application 3.2. Consider the Stancu univariate operator based on factorial powers $P_{m}^{\langle\alpha\rangle}$ : $C[0,1] \rightarrow C[0,1]$ given by

$$
P_{m}^{\langle\alpha\rangle}(f ; x)=\sum_{k=0}^{m} p_{m, k}^{\langle\alpha\rangle}(x) f\left(\frac{k}{m}\right),
$$

where $\alpha=\alpha(m) \geq 0$ and

$$
p_{m, k}^{\langle\alpha\rangle}=\left(\begin{array}{c}
m \\
k
\end{array}\right) \frac{x^{[k,-\alpha]}(1-x)^{[m-k,-\alpha]}}{1^{[m,-\alpha]}} .
$$

In [26] Miclăuş proved that if, $f$ is convex of 1-th order on [0,1], the sequence $\left\{P_{m}^{\langle\alpha\rangle}(f ; x)\right\}_{m \in \mathbb{N}}$ is monotonous decreasing and $P_{m}^{\langle\alpha\rangle}(f ; x)>f(x),(\forall) x \in[0,1]$. Using the method of parametric extension, Miclăuş [27] constructed the bivariate Stancu operator based on factorial powers $P_{m, n}^{\langle\alpha, \beta\rangle}: C([0,1] \times[0,1]) \rightarrow C([0,1] \times[0,1])$, given by 


$$
P_{m, n}^{\langle\alpha, \beta\rangle}(f ; x, y)=\sum_{k=0}^{m} \sum_{j=0}^{n} p_{m, k}^{\langle\alpha\rangle}(x) p_{n, j}^{\langle\beta\rangle}(y) f\left(\frac{k}{m}, \frac{j}{n}\right) .
$$

In the same paper [27] was studied the remainder term of the bivariate Stancu approximation formula

$$
f=P_{m, n}^{\langle\alpha, \beta\rangle}(f)+R_{m, n}^{\langle\alpha, \beta\rangle}(f),
$$

and was obtained an representation of the remainder term using bivariate divided differences.

Let now $\left(x_{0}, y_{0}\right) \in[0,1] \times[0,1]$ arbitrarily chosen and define the linear functional $A \in$ $C^{\#}([0,1] \times[0,1])$ by

$$
A(f)=f\left(x_{0}, y_{0}\right)-P_{m, n}^{\langle\alpha, \beta\rangle}\left(f ; x_{0}, y_{0}\right) .
$$

From (3.70) we get the following:

(i) $A\left(x_{0}^{i} y_{0}^{j}\right)=0$, for $i \in\{0,1\}, j \in\{0,1\}$;

(ii) $A\left(x_{0}^{2} y_{0}^{2}\right) \neq 0$;

(iii) $A(g) \neq 0$ for any $g \in C([0,1] \times[0,1])$ convex of $(1,1)$-th order.

Consequently, from Theorem 3.2 it follows there exist the distinct points $\left(\xi_{i} \eta_{j}\right) \in[0,1] \times$ $[0,1], i \in\{1,2,3\}, j \in\{1,2,3\}$ such that

$$
A(f)=A\left(x_{0}^{2} y_{0}^{2}\right)\left[\begin{array}{c}
\xi_{1}, \xi_{2}, \xi_{3} \\
\eta_{1}, \eta_{2}, \eta_{3}
\end{array} ; f\right]
$$

Taking into account that

$$
A\left(x_{0}^{2} y_{0}^{2}\right)=\frac{1+\alpha m}{m(1+\alpha)} \cdot \frac{1+\beta n}{n(1+\beta)} x_{0} y_{0}\left(1-x_{0}\right)\left(1-y_{0}\right)\left[\begin{array}{c}
\xi_{1}, \xi_{2}, \xi_{3} \\
\eta_{1}, \eta_{2}, \eta_{3}
\end{array} ; f\right],
$$

and $\left(x_{0}, y_{0}\right) \in[0,1] \times[0,1]$ was arbitrarily chosen, we get that the remainder term of (3.69) can be expressed under the form

$$
R_{m, n}^{\langle\alpha, \beta\rangle}(f ; x, y)=\frac{1+\alpha m}{m(1+\alpha)} \cdot \frac{1+\beta n}{n(1+\beta)} x y(1-x)(1-y)\left[\begin{array}{c}
\xi_{1}, \xi_{2}, \xi_{3} \\
\eta_{1}, \eta_{2}, \eta_{3}
\end{array} ; f\right],
$$

for any $(x, y) \in[0,1] \times[0,1]$, where $0 \leq \xi_{1}<\xi_{2}<\xi_{3} \leq 1$ and $0 \leq \eta_{1}<\eta_{2}<\eta_{3} \leq 1$.

Remark 3.5. In the case when $\alpha=\beta=0$ one obtains the Bernstein bivariate approximation formula (3.57) and (3.73) is reduced to (3.65).

Application 3.3. Let $p \in \mathbb{N}_{0}$. In 1962, Schurer in [33] introduced the linear operator $\widetilde{B}_{m, p}$ : $C([0,1+p]) \rightarrow C([0,1])$, given by

$$
\widetilde{B}_{m, p}(f ; x)=\sum_{k=0}^{m+p} \widetilde{p}_{m, k}(x) f\left(\frac{k}{m}\right),
$$

where $\widetilde{p}_{m, k}(x)$ are the fundamental Schurer's polynomials, defined by

$$
\widetilde{p}_{m, k}(x)=\left(\begin{array}{c}
m+p \\
k
\end{array}\right) x^{k}(1-x)^{m+p-k} .
$$


Note that the operator (3.74) is positive only on $C[0,1]$ and the sequence $\left\{\widetilde{B}_{m, p}(f)\right\}_{m \in \mathbb{N}}$ convergences uniformly to $f \in C[0,1+p]$ only on $[0,1]$.

Muraru [28] proved that if $f \in C[0,1+p]$ is convex of 1-th order, the sequence of Schurer's polynomials is monotonous decreasing and $B_{m}(f ; x)>f(x)$, for any $x \in[0,1]$.

More at this, taking into account that

$$
\begin{aligned}
& \widetilde{B}_{m, p}(1 ; x)=1, \\
& \widetilde{B}_{m, p}(t ; x)=\left(1+\frac{p}{m}\right) x,
\end{aligned}
$$

it follows (applying Theorem 3.1) that the remainder term of the Schurer approximation formula

$$
f=\widetilde{B}_{m, p}(f)+\widetilde{R}_{m, p}(f),
$$

can be represented under the form

$$
\widetilde{R}_{m, p}(f)=-\frac{m+p}{x}\left[\xi_{1}, \xi_{2} ; f\right]
$$

where $0 \leq \xi_{1}<\xi_{2}<\xi_{3} \leq 1$.

In [7], using the method of parametric extensions [21], [5] was constructed the bivariate Schurer operator $\widetilde{B}_{m, p, n, q}: C([0,1+p] \times[0,1+q]) \rightarrow C([0,1+p] \times[0,1+q])$, given by

$$
\widetilde{B}_{m, p, n, q}(f ; x, y)=\sum_{k=0}^{m+p} \sum_{j=0}^{n+q} \widetilde{p}_{m, p}(x) \widetilde{p}_{n, q}(y) f\left(\frac{k}{m}, \frac{j}{n}\right) .
$$

It is immediately that $\widetilde{B}_{m, p, n, q}$ is monotonous decreasing on $[0,1] \times[0,1]$ for each $f \in C([0,1+$ $p] \times[0,1+q])$ monotonous strictly increasing and $\widetilde{B}_{m, p, n, q}(f ; x, y)>f(x, y)$ for any $(x, y) \in$ $[0,1] \times[0,1]$.

Consequently, the remainder term of the Schurer bivariate approximation formula

$$
f=\widetilde{B}_{m, p, n, q}(f ; x, y)+\widetilde{R}_{m, p, n, q}(f ; x, y),
$$

can be expressed under the form

$$
\widetilde{R}_{m, p, n, q}(f ; x, y)=\left(1+\frac{p}{n}\right)\left(1+\frac{q}{n}\right) x y\left[\begin{array}{c}
\xi_{1}, \xi_{2} \\
\eta_{1}, \eta_{2}
\end{array} ; f\right],
$$

for any $(x, y) \in[0,1] \times[0,1]$, where $0 \leq \xi_{1}<\xi_{2} \leq 1$ and $0 \leq \eta_{1}<\eta_{2} \leq 1$.

\section{REFERENCES}

[1] A. M. Acu, Properties and applications of $P_{n}$-simple functionals, Positivity, 21 (2017), No. 1, 283-297

[2] U. Abel, and M. Ivan, New representation of the remainder in the Bernstein approximation, J. Math. Anal. Appl., 381 (2011), No. 2, 952-956

[3] O. Agratini, Approximation by linear operators (in Romanian), Presa Universitară Clujeană, Cluj-Napoca, 2000

[4] O. Aramă, Proprietăţi privind monotonia şirului polinoamelor de interpolare ale lui S. N. Bernstein şi aplicarea lor la studiul aproximării funcţiilor , Studii şi Cerc. Mat., 8 (1957), 195-210

[5] D. Bărbosu, Aproximarea funcţiilor de mai multe variabile prin sume booleene de operatori liniari de tip interpolator, Ed. Risoprint, Cluj-Napoca, 2002 (in Romanian)

[6] D. Bărbosu, Schurer-Stancu type operators, Studia Univ. "Babeş-Bolyai" Math., 48 (2003), No. 3, 31-35 
D. Bărbosu

[7] D. Bărbosu, Bivariate operators of Schurer-Stancu type, An. Ştiinţ. Univ. Ovidius Constanța Ser. Mat., 11 (2003), No. $1,1-8$

[8] D. Bărbosu, GBS operators of Schurer-Stancu type, An. Univ. Craiova Ser. Mat. Inform., 30 (2003), No. 2, 34-39

[9] D. Bărbosu, Polynomial approximation by means of Schurer-Stancu type operators, Ed. Univ. de Nord, Baia Mare, 2006

[10] D. Bărbosu, Two dimensional divided differences revisited, Creat. Math. Inform., 17 (2008), 1-7

[11] D. Bărbosu and O. T. Pop, A note on the GBS Bernstein's approximation formula, An. Univ. Craiova Ser. Mat. Inform., 35 (2008), 1-6

[12] D. Bărbosu and O. T. Pop, On the Bernstein bivariate approximation formula , Carpathian J. Math., 24 (2008), No. 3 , 293-298

[13] D. Bărbosu and O. T. Pop, Bivariate Schurer-Stancu operators revisited, Carpathian J. Math., 26 (2010), No. 1, 24-35

[14] D. Bărbosu, On the monotonicity of Schurer-Stancu's polynomials, Automat. Comput. Appl. Math., 15 (2006), No. 1, 27-35

[15] D. Bărbosu and D. Miclăuş, On the Stancu operators and their applications, Creat. Math. Inform., 26 (2017), No. 1, 29-36

[16] D. Bărbosu, On the approximation of convex functions using linear positive operators, Creat. Math. Inform., 26 (2017), No. 2, 137-143

[17] D. Bărbosu, On the monotonicity of bivariate Bernstein polynomials, Creat. Math. Inform., 27 (2018), No. 1, 9-14

[18] S. N. Bernstein, Démonstration du théorème de Weierstrass fondée sur le calcul de probabilités, Commun. Soc. Math. Kharkow, 13 (1912-1913), No. 2, 1-2

[19] V. Gupta, Differences of Operators of Lupaş Type, Constructive Mathematical Analysis 1 (1) (2018), 9-14

[20] B. Della Vecchia, On the approximation of functions by means of the operators of D. D. Stancu, Studia Univ. Babeş-Bolyai, Mathematica, 37 (1992), No. 1, 3-36

[21] F. J. Delvos and W. Schempp, Boolean methods in interpolation and approximation, Pitman Research Notes in Math., Series 230, New York, 1989

[22] D. V. Ionescu, Divided differences (in Romanian), Ed. Acad. R.S.R., Bucureşti, 1978

[23] M. Ivan, Elements of Interpolation Theory, Mediamira Science Publisher, Cluj-Napoca (2004), 61-68

[24] D. Miclăuş, The revision of some results for Bernstein-Stancu type operators, Carpathian J. Math., 28 (2012), No. 2 , 289-300

[25] D. Miclăuş, On the GBS Bernstein-Stancu's type operators, Creat. Math. Inform., 22 (2013), No. 1, 73-80

[26] D. Miclăuş, On the monotonicity property for the sequence of Stancu type polynomials, An. Ştiinţ. Univ. Al. I. Cuza Iaşi. Mat. (N.S.), 62 (2016), No. 1, 141-149

[27] D. Miclăuş, On the Stancu type bivariate approximation formula, Carpathian J. Math., 32 (2016), No. 1, 103-111

[28] C. Muraru, On the monotonicity of Schurer type polynomials, Carpathian J. Math., 21 (2005), No. 1-2, 89-94

[29] O. T. Pop and D. Bărbosu, Two dimensional divided differences with multiple knots, An. Ştiinţ. Univ. "Ovidius" Constanţa Ser. Mat.,17 (2009), No. 2, 181-190

[30] T. Popoviciu, Sur quelques proprietes des fonctions d'une ou deux variables réelles, Mathematica (1934), 1-85

[31] T. Popoviciu, Introduction à la théorie des différences divisées, (French) Bull. Math. Soc. Roumaine Sci., 42 (1940), No. $1,65-78$

[32] R. T. Rockafeller, Convex Analysis, Ed. Theta, Bucharest, 1992 ( in Romanian, translated by Ingrid and Daniel Beltiţă )

[33] F. Schurer, Linear positive operators in approximation theory, Math. Inst. Tech. Univ Delft Report, 1962

[34] D. D. Stancu, Some Bernstein poiynomials in two variables and their applications,Soviet Math. Dokl., 1 (1961), 1025-1028

[35] D. D. Stancu, The remainder of certain linear approximation formulas in two variables, J. SIAM Numer. Anal., 1 (1964), 137-163

[36] D. D. Stancu, Approximation of functions by a new class of linear polynomial operators, rev. Roum. Math. Pures et Appl., 13 (1968), No. 8, 1173-1194

[37] D. D. Stancu, Asupra unei generalizări a polinoamelor lui Bernstein (Romanian), Studia Univ. "Babeş-Bolyai", Ser. Mathematica-Physica, (1969), No. 2, 31-45

[38] D. D. Stancu, On the remainder of approximation of functions by means of a parameter-dependent linear polynomial operator, Studia Univ. Babeş-Bolyai Ser. Math.-Mech., 16 (1971), No. 2, 59-66

[39] D. D. Stancu, Application of divided differences to the study of monotonicity of the derivatives of the sequence of Bernstein polynomials, Calcolo 16 (1979), No. 4, 431-445

[40] D. D. Stancu, Gh. Coman, O. Agratini and R. Trâmbiţaş, Analiză numerică şi teoria aproximării, vol. I, Presa Universitară Clujeană, Cluj-Napoca, 2001 (in Romanian) 
TECHNICAL UNIVERSITY OF CLUJ-NAPOCA

North University CENTER AT BAIA MARE

DePARTMENT OF MATHEMATICS AND COMPUTER SCIENCE

VICTORIEI 76, 430122 BAIA MARE, ROMANIA

E-mail address: barbosudan@yahoo.com 